\title{
The second Born approximation of electron-argon elastic scattering in a Bichromatic laser field
}

\author{
Bin Zhou ${ }^{1}$, Ming-Yang Zheng ${ }^{2}$ and Da-Yong Wen ${ }^{3}$ \\ ${ }^{1}$ Physics lab, nanxu college, Jiangsu University of Science and Technology, \\ Zhenjiang, Jiangsu 212003, People's Republic of China \\ ${ }^{2}$ Department of Modern Physics, University of Science and Technology of China, \\ Hefei, Anhui 230026, People's Republic of China \\ ${ }^{3}$ Nanxu college, Jiangsu University of Science and Technology, \\ Zhenjiang, Jiangsu 212003, People's Republic of China.
}

(Dated: January 19, 2021)

\begin{abstract}
We study the elastic scattering of atomic argon by electron in the presence of a bichromatic laser field in the second Born approximation. The target atom is approximated by a simple screening potential and the continuum states of the impinging and emitting electrons are described as Volkov states. We evaluate the S-matrix elements numerically. The dependence of differential cross section on the relative phase between the two laser components is presented. The results obtained in the first and second Born approximation are compared and analysed.
\end{abstract}

PACS numbers: 34.80.Qb; 32.80.Wr; 34.50.Rk; 34.80.Bm

The process of multiphoton free-free transitions (MFFT) was firstly studied by Bunkin and Fedorov [1], and has attracted much attention in physical community. Summaries of these investigations were presented in the book by Mittleman [2] and some reviews [3, 4]. The theoretical work treating the laser radiation classically with a single frequency $\omega$, or some narrow band multi-mode approximation has yielded prefect agreement with the experiments by Weigngarshofer [5]. With the development of laser technology, the atomic and molecular processes assisted or induced by powerful and new kinds of laser fields have been researched, especially for the case by multicolor laser. Free-free transitions in a powerful bichromatic laser field has become feasible experimentally to coherently control the phase between the two components of the radiation field. A considerable body of research work has concentrated on the coherent phase control (CPC) of the elastic stattering processes [6-18]. In such mentioned papers, the multiphoton processes were treated in the first Born approximation (FBA), while in this letter, we carried out the calculation in the second-order Born approximation (SBA) and compared the results with that in the FBA formation. Atomic units $\hbar=m=e=1$ are used throughout.

Considering that in a laser beam the density of radiation quanta is so large that the depletion of this beam by emitting or absorbing quanta from it is negligible, then the laser field is treated classically in our model. Hence, the bichromatic laser field is described as a classical electromagnetic field with the fundamental frequency $\omega$ and its second harmonic $2 \omega$, i.e., $\mathcal{E}(t)=$ $\mathcal{E}_{\circ}[\sin \omega t+\sin (2 \omega t+\varphi)]$, where $\mathcal{E}_{\text {。 }}$ is the electric field amplitude and the relative phase $\varphi$ can be arbitrarily changed.

The target atom is described by a screening potential
[19]:

$$
V(r)=-\frac{\mathrm{Z}}{r} \sum_{i=1}^{3} A_{i} \exp \left(-\alpha_{i} r\right),
$$

where $r$ denotes the position of the electron with respect to the nucleus, and $\mathrm{Z}$ is the nuclear charge number. For argon, $A_{1}=2.1912, A_{2}=-2.8252, A_{3}=1-A_{1}-A_{2}$, $\alpha_{1}=5.5470, \alpha_{2}=4.5687$, and $\alpha_{3}=2.0446$.

The scattering matrix for the laser-assisted free-free transition in the second Born approximation reads:

$$
S_{f i}^{<2>}=S_{f i}^{(1)}+S_{f i}^{(2)},
$$

where

$$
S_{f i}^{(1)}=-i\left\langle\chi_{\boldsymbol{k}_{f}}|V| \chi_{\boldsymbol{k}_{i}}\right\rangle .
$$

Here $\chi_{\boldsymbol{k}_{i}}$ and $\chi_{\boldsymbol{k}_{f}}$ are the states of the electrons in the initial and final channels, described by the Volkov wave function:

$$
\begin{aligned}
\chi_{\boldsymbol{k}_{i, f}}= & \exp \left(i \boldsymbol{k}_{i, f} \cdot r\right) \exp \left[-i E_{i, f} t-\frac{i}{\omega^{2}} \boldsymbol{k}_{i, f} \cdot \mathcal{E}_{\circ} \sin \omega t\right] \\
& \exp \left[-\frac{i}{4 \omega^{2}} \boldsymbol{k}_{i, f} \cdot \mathcal{E}_{\circ} \sin (2 \omega t+\varphi)\right],
\end{aligned}
$$

where $\boldsymbol{k}_{i, f}$ are the wave vectors of incident and scattered electrons, and $E_{i, f}$ are the corresponding kinetic energies.

Using the potential of Eq. (11) and the wave functions in Eq. (44), the first term of the $S_{f i}^{<2>}$ can be recast as:

$$
S_{f i}^{(1)}=-2 \pi i \sum_{l} T_{f i}^{(1)}(l) \delta\left(E_{f}-E_{i}+l \omega\right) .
$$

$T_{f i}^{(1)}(l)$ is the ionization amplitude accompanying the exchange of $l$ photons with the laser field $(l>0$ for emission, and $l<0$ for absorption),

$$
T_{f i}^{(1)}(l)=B_{l}\left(\lambda, \frac{1}{4} \lambda, \varphi\right) V\left(\boldsymbol{k}_{f, i}\right),
$$



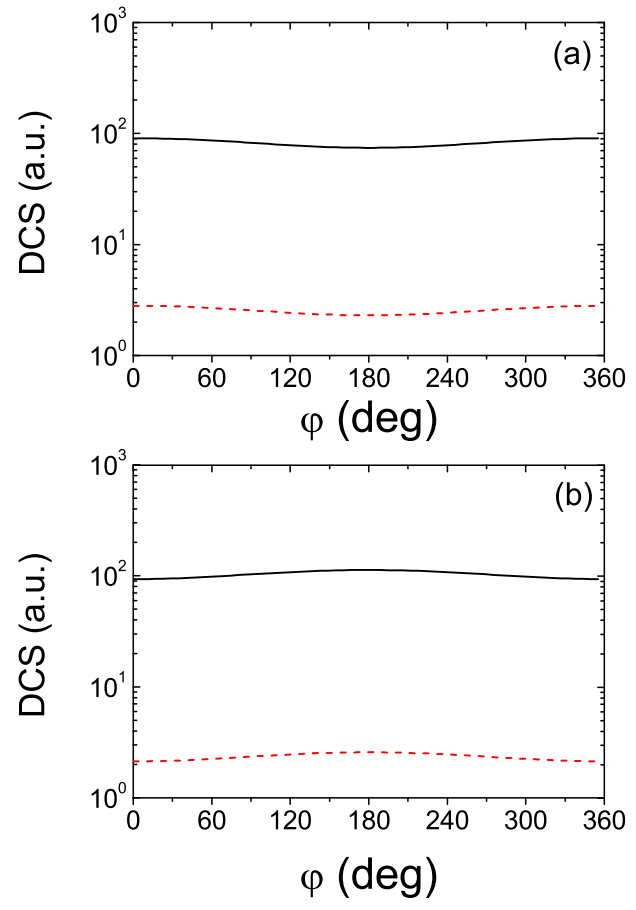

Fig. 1: (a) The DCS versus $\varphi$ for elastic electron-argon scattering with emission of one photon $(l=1)$. The scattered angle of emitting electron is $\theta=13^{\circ}$. The kinetic energies of the incident electron is $E_{i}=9.5 \mathrm{eV}$. Solid curve: SBA results; dashed curve: FBA results. (b) The same as (a), but for the case with one photon absorption $(l=-1)$.

in which

$$
V\left(\boldsymbol{k}_{f, i}\right)=\int d \boldsymbol{r} e^{-\mathrm{i}\left(\boldsymbol{k}_{f}-\boldsymbol{k}_{i}\right) \cdot \boldsymbol{r}} V(r)
$$

Clearly it is just the Fourier transformation of the potential. The other term has the form:

$$
B_{l}\left(\lambda, \frac{1}{4} \lambda, \varphi\right)=\sum_{n=-\infty}^{\infty} J_{l-2 n}(\lambda) J_{n}\left(\frac{1}{4} \lambda\right) \exp (-\mathrm{i} n \varphi)
$$

which is the generalized Bessel function with $\lambda=\left(\boldsymbol{k}_{f}-\right.$ $\left.\boldsymbol{k}_{i}\right) \cdot \mathcal{E}_{0} / \omega^{2} . J_{n}$ is the ordinary Bessel function.

The second term of SBA matrix is:

$$
\begin{gathered}
S_{f i}^{(2)}=-i \int d \boldsymbol{r} \int d \boldsymbol{r}^{\prime} \int_{-\infty}^{\infty} d t \int_{-\infty}^{\infty} d t^{\prime} \chi_{\boldsymbol{k}_{f}}^{*}(\boldsymbol{r}, t) \\
V(r) G\left(\boldsymbol{r}, t ; \boldsymbol{r}^{\prime}, t^{\prime}\right) V\left(r^{\prime}\right) \chi_{\boldsymbol{k}_{i}}\left(\boldsymbol{r}^{\prime}, t\right),
\end{gathered}
$$

where $G\left(\boldsymbol{r}, t ; \boldsymbol{r}^{\prime}, t^{\prime}\right)$ is the Green function:

$$
G\left(\boldsymbol{r}, t ; \boldsymbol{r}^{\prime}, t^{\prime}\right)=-\frac{i}{(2 \pi)^{3}} \int d \boldsymbol{k} \chi_{\boldsymbol{k}}(\boldsymbol{r}, t) \chi_{\boldsymbol{k}}^{*}(\boldsymbol{r}, t) u\left(t-t^{\prime}\right) .
$$
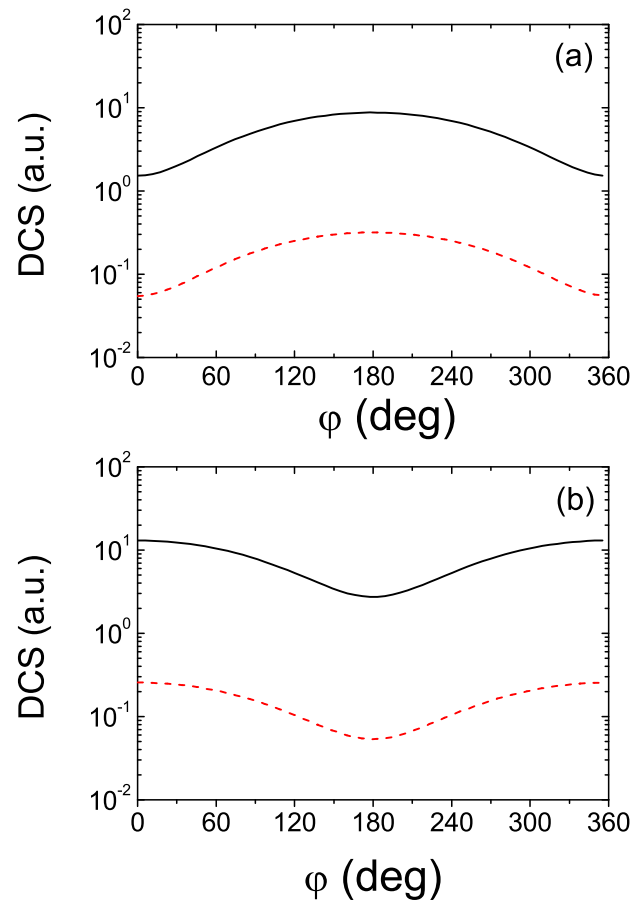

Fig. 2: (a) The parameters are the same as Fig. 1 except for the number of the exchanged photons. For (a), $l=2$, while for $(b), l=-2$. Solid curve: SBA results; dashed curve: FBA results.

Here $u\left(t-t^{\prime}\right)$ is the step function.

Using Eq. (4) and Eq. (9), we obtain:

$$
S_{f i}^{(2)}=-2 \pi i \sum_{l} T_{f i}^{(2)}(l) \delta\left(E_{f}-E_{i}+l \omega\right),
$$

where the photon-number-resolved transition amplitude can be expressed as:

$$
\begin{aligned}
T_{f i}^{(2)}(l)= & \frac{1}{(2 \pi)^{3}} \sum_{m} \int d \boldsymbol{k} \frac{1}{E_{i}-E-m \omega+i \eta} \\
& V\left(\boldsymbol{k}_{f}, \boldsymbol{k}\right) V\left(\boldsymbol{k}, \boldsymbol{k}_{i}\right) B_{m}\left(\lambda_{1}, \frac{1}{4} \lambda_{1}, \varphi\right) \\
& B_{l-m}\left(\lambda_{2}, \frac{1}{4} \lambda_{2}, \varphi\right),
\end{aligned}
$$

where $\eta$ is a small positive quantity.

So we can obtain the SBA scattering amplitude:

$$
T_{f i}^{<2>}(l)=T_{f i}^{(1)}(l)+T_{f i}^{(2)}(l) .
$$

The differential cross sections (DCS) for the net exchange of $l$ photons between the colliding system and the bichromatic laser field can be described as:

$$
\frac{d \sigma}{d \Omega}=\left(\frac{1}{2 \pi}\right)^{2} \frac{k_{f}}{k_{i}}\left|T_{f i}^{<2>}(l)\right|^{2} .
$$



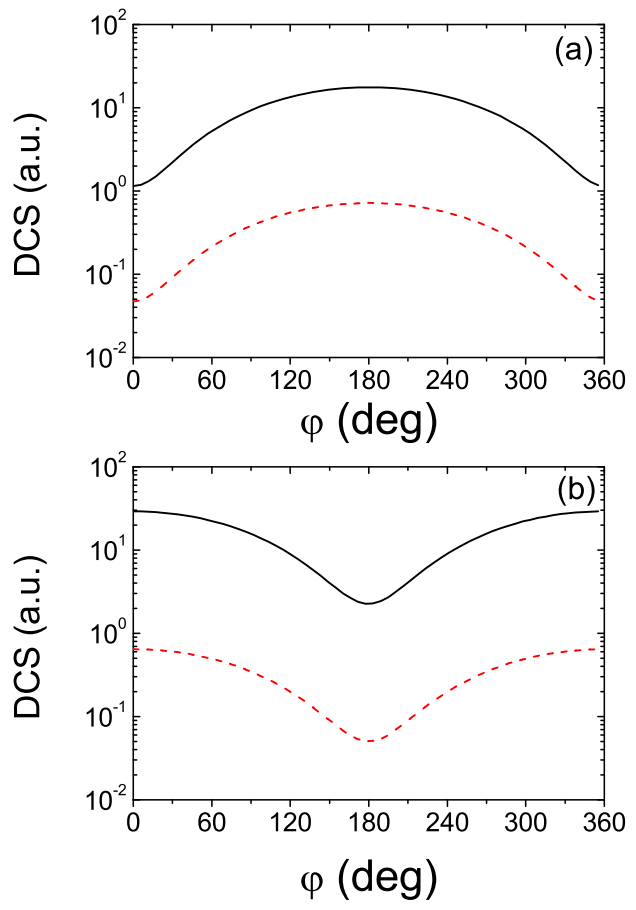

Fig. 3: The parameters are the same as Fig. 2 except for the impact energy. In this figure, the energy of the incoming electron is $E_{i}=19.5 \mathrm{eV}$. Solid curve: SBA results; dashed curve: FBA results.

For numerical calculation, we studied the dependence of differential cross section for electron-argon atom scattering on the relative phase angle $\varphi$ between the two laser components under the geometry of the experiment by Weingartshofer [5]. The angle between the polarization vector $\mathcal{E}$ and the momentum of the incident electron $\mathbf{k}_{i}$ is $\phi=38^{\circ}$, the momentum $\mathbf{k}_{f}$ of the scattered electron is in the plane defined by the polarization vector $\mathcal{E}$ and $\mathbf{k}_{i}$. The bichromatic laser parameters are $\omega=0.117 \mathrm{eV}$ and $\mathcal{E}_{0}=2.7 \times 10^{8} \mathrm{~V} / \mathrm{cm}$.

In Fig. 1, we show the DCS versus the phase angle $\varphi$. The $\mathrm{CPC}$ effects are not very apparent for one photon exchange process. The distribution of the controlling effect is centered on $\varphi=180^{\circ}$. Such phenomenon is more distinct for the processes with more than one photons transfer which could be seen clearly in the following two figures. This may attribute to the fact that the generalized Bessel function satisfies the relationship [4]: $B_{-l}(a, b, \varphi)=(-1)^{l} B_{l}^{*}(a, b, \varphi-\pi)$. Moreover, the results have some prominent improvements in the second Born approximation. This indicates that the intermediat states have significant contribution to the collision processes.
Figure 2 displays the DCS versus $\varphi$ for two photons transfer. As is depicted in the figure, the DCS for $l= \pm 2$ are much smaller than $l= \pm 1$. This demonstrates that the probability of two photons transfer between laser field and the colliding system is small. With the increase of $|l|$ (not presented here), the DCS becomes smaller and smaller. For the case with two photons emission $(l=2)$, more electrons are emitted at $\theta=13^{\circ}$ when the phase difference between the laser components is near $\varphi=180^{\circ}$ where the CPC effect reaches the maximum; while for $l=$ -2 , the situation is inverse. The second-order corrections are still distinct, and for different $\varphi$, the contribution of the intermediate states are almost the same.

In Fig. 3, we show the influence of the impact energy on the collision process. It is evident that the DCS becomes larger when the energy of the incident electron increases and the CPC effects are more prominent.

In summary, the electron-argon elastic scattering in a bichromatic laser field is investigated in the second Born approximation. The dependence of DCS on the relative phase is analysed. The DCS are significantly improved in the second Born approximation. We attribute it to the contributions of the intermediate states.

This work is supported by the National Nature Science Foundation of China under Grants No. 10874169 and No. 10674125, and the National Basic Research Program of China under Grants No. 2007CB925200 and No. 2010 CB923301.

[1] F. V. Bunkin and M. V. Fedorov, Sov. Phys. Rep 22, 844 (1996).

[2] M. H. Mittleman, Introduction to the Theroy for LaserAtom Interactions. Plenum. New York (1982).

[3] C. J. Joachain, M. Dörr and N. J. Kylstra, Adv. At. Mol. Opt. Phys 42, 225 (2000).

[4] F. Ehlotzky, Physics. Reports 345, 175 (2001).

[5] A. Weingartshofer, J. K. Holemes, J. Sabbagh, and S. L. Chin, J. Phys. B 16, 1805 (1983).

[6] J. F. Sun, M. C. Liang, and Z. L. Zhu, Chin. Phys. Lett 242572 (2007)

[7] D. Nehari, J. Holmes, K. M. Dunseath, and M. TeraoDunseath, J. Phys. B 43, 025203 (2010)

[8] B. A. Harak, L. Ladino, and N. L. S Martin, Bulletin of the American Physical Society 55, 5 (2010)

[9] M. Ghalim and F. Mastour, J. Phys. B 32, 3783 (1999).

[10] S. T. Zhang, J. Chen, and S. M. Li, Can. J. Phys 80, 969 (2002).

[11] M. Protopapas, P. L. Knight, and K. Burnett, Phys. Rev. A 49, 1945 (1994).

[12] J. Z. Kamiński and F. Ehlotzky, Phys. Rev. A 50, 4404 (1994).

[13] S. Varró and F. Ehlotzky, J. Phys. B 28, 1613 (1995).

[14] S. T. Zhang and J. Chen, J. Phys. B 34, 2201 (2001).

[15] S. Varró and F. Ehlotzky, J. Phys. B 30, 1061 (1995).

[16] S. Varró and F. Ehlotzky, Phys. Rev. A 47, 715 (1993).

[17] A. Cionga, F. Ehlotzky, and G. Zloh, J. Phys. B 34, 2057 
(2001).

[18] D. B. Miloševic and F. Ehlotzky, Phys. Rev. A 56, 3879 (1997).
[19] F. Salvat, J. D. Martínez, R. Mayol, and J. Parellada, Phys. Rev. A 36, 467 (1987). 